\title{
Novel COL4A1 mutations identified in infants with congenital hemolytic anemia in association with brain malformations
}

\author{
Hiromi Ogura', Shouichi Ohga², Takako Aoki', Taiju Utsugisawa', Hidehiro Takahashi ${ }^{3}$, Asayuki Iwai', \\ Kenichiro Watanabe ${ }^{5}$, Yusuke Okuno ${ }^{6}$, Kenichi Yoshida $0^{7}$, Seishi Ogawa ${ }^{7,8,9}$, Satoru Miyano ${ }^{10}$, Seiji Kojima ${ }^{11}$, \\ Toshiyuki Yamamoto (id ${ }^{12}$, Keiko Yamamoto-Shimojima, ${ }^{1,12}$ and Hitoshi Kanno (i)
}

\begin{abstract}
Genetic causes of undiagnosed hemolytic anemia in nineteen patients were analyzed by whole-exome sequencing, and novel COLAA1 variants were identified in four patients (21\%). All patients were complicated with congenital malformations of the brain, such as porencephaly or schizencephaly. In these patients, hemolysis became less severe within 2 months after birth, and red cell transfusion was no longer required after 50 days, whereas chronic hemolysis continued.
\end{abstract}

Congenital hemolytic anemia is caused by genetic abnormalities of the red cell membrane, enzyme, or hemoglobin. We have been performing research studies on congenital hemolytic anemia. As a result, extensive analyses of hemoglobin, red cell membrane, and enzymes have been performed. However, approximately $40 \%$ of patients remained undiagnosed (personal data). Recently, we encountered infantile patients with hemolytic anemia in whom novel COLAA1 variants were identified. Here, detailed information on the patients and the identified variants is reported.

This study was performed in accordance with the Declaration of Helsinki and was approved by the Ethics Committee of the institution. After receiving written an informed consent from the patients' families, we obtained blood samples from patients and their parents. Genomic DNA was extracted using a standard protocol. Using trio samples, whole-exome sequencing (WES) was performed,

Correspondence: Keiko Yamamoto-Shimojima (shimojima.keiko@twmu.ac.jp) 'Department of Transfusion Medicine and Cell Processing, Tokyo Women's Medical University, Tokyo, Japan

${ }^{2}$ Department of Pediatrics, Graduate School of Medical Sciences, Kyushu University, Fukuoka, Japan

Full list of author information is available at the end of the article as described previously ${ }^{1}$. The extracted data were mapped to a reference genome (GRCh37/hg19), annotated, and filtered in accordance with the method described previously ${ }^{1}$. The possible candidate variants were reconfirmed by Sanger sequencing.

Nineteen infants with undiagnosed congenital hemolytic anemia were included in this study. Immunological hemolysis, unstable hemoglobinopathies, red cell membrane defects and red cell enzymopathies were ruled out by routine laboratory tests, including direct a antiglobulin test, isopropanol test, osmotic fragility test, eosin $5^{\prime}$-maleimide binding test, acidified glycerol lysis time, and reduced glutathione and 15 red blood cell enzyme activities.

Through WES, four novel COL4A1 missense variants were identified among 19 patients with undiagnosed infantile hemolytic anemia $(4 / 19=21 \%)$ : NM_001845.6: c.2354G $>$ T [p.Gly785Val], c.2537G $>$ A [p.Gly846Glu], c.2788G >A [p.Gly930Ser] and c.2843G $>$ A [p.Gly948Asp] (Table 1). In the WES data, there were no other possible candidate variants related to hemolytic anemia. The identified COL4A1 variants were reconfirmed by Sanger sequencing. According to the ACMG recommendation, 
Table 1 Results of this study.

\begin{tabular}{|c|c|c|c|c|}
\hline & Case 1 & Case 2 & Case 3 & Case 4 \\
\hline \multirow[t]{3}{*}{ Mutation } & chr13:110831374 & chr13:110830500 & chr13:110829313 & chr13:110829258 \\
\hline & NM_001845.6:c.2354G>T & NM_001845.6:c.2537G>A & NM_001845.6:c.2788G>A & NM_001845.6:c.2843G>A \\
\hline & p.Gly785Val & p.Gly846Glu & p.Gly930Ser & p.Gly948Asp \\
\hline CADD_phred & 23.1 & 24.1 & 35 & 24.3 \\
\hline ACMG criteria & PS1, PS2, PM2, PP3, and PP4 & PS2, PM2, PP3, and PP4 & PS2, PM2, PP3, and PP4 & $\begin{array}{l}\text { PS2, PM2, PM5, PP3, } \\
\text { and PP4 }\end{array}$ \\
\hline Interpretation & Pathogenic & Likely pathogenic & Likely pathogenic & Pathogenic \\
\hline Inheritance & de novo & de novo & de novo & de novo \\
\hline Gender & M & $\mathrm{F}$ & $\mathrm{F}$ & $\mathrm{F}$ \\
\hline Age (month) & 0 & 2 & 2 & 3 \\
\hline Family history & - & - & - & - \\
\hline $\begin{array}{l}\text { Period of } \\
\text { gestation (week) }\end{array}$ & 39 & 37 & 35 & 37 \\
\hline Birth weight (g) & 2190 & 2467 & 1624 & 2336 \\
\hline Test day (day) & 0 & 47 & 81 & 115 \\
\hline $\mathrm{Hb}(\mathrm{g} / \mathrm{dL})$ & 9.3 & 6.8 & 6.7 & 7.4 \\
\hline $\mathrm{MCV}(\mathrm{fL})$ & 127 & 99 & 110 & 90 \\
\hline MCHC (\%) & 31.4 & 31.9 & 29.9 & 32.7 \\
\hline Retics (\%) & 16.4 & 9.2 & 14 & ND \\
\hline $\mathrm{LDH}(\mathrm{U} / \mathrm{L})$ & 582 & 302 & 242 & 847 \\
\hline $\mathrm{Hp}(\mathrm{mg} / \mathrm{dL})$ & 1 & $<10$ & $<7.1$ & $<10$ \\
\hline Osmotic fragility & ND & ND & ND & - \\
\hline EMA (\% of Ct) & ND & 87.6 & 98.1 & 94.2 \\
\hline RBC morphology & Normal & Poikilocytes & Shizocytes & Anisocytes \\
\hline $\begin{array}{l}\text { Icterus gravis } \\
\text { neonatorum }\end{array}$ & + & - & + & + \\
\hline $\begin{array}{l}\text { Last blood transfusion } \\
\text { date (day) }\end{array}$ & 6 & 50 & 45 & 51 \\
\hline Splenomegaly & - & - & - & - \\
\hline Phototherapy & + & - & + & + \\
\hline $\begin{array}{l}\text { Exchange blood } \\
\text { transfusion }\end{array}$ & 7 times & - & - & - \\
\hline Improvement of anemia & + & + & + & + \\
\hline Others & $\begin{array}{l}\text { Microcephaly, paraventricular } \\
\text { calcification, cataract }\end{array}$ & $\begin{array}{l}\text { Schizencephaly, cataract, } \\
\text { macroglossia }\end{array}$ & $\begin{array}{l}\text { Microcephaly, porencephaly with } \\
\text { paraventricular carcification }\end{array}$ & $\begin{array}{l}\text { Hydrocephaly, } \\
\text { epidermolysis bullosa }\end{array}$ \\
\hline
\end{tabular}

EMA eosin 5'-maleimide, ND not detected

all variants were evaluated as "pathogenic" or "likely pathogenic" 2 .

Clinical information on the patients is also summarized in Table 1. All patients were born with low birth weight in association with moderate to severe neonatal hemolytic anemia. The prenatal medical histories of all patients were not remarkable. Morphological changes in the peripheral blood smear were observed in three patients (Cases 2, 3, and 4). All patients showed spontaneous remission of hemolytic anemia within 2 months after birth and no longer required red cell transfusion after 50 days. All patients were complicated with congenital anomalies in 
the central nervous system, such as porencephaly or schizencephaly.

In 2005, COL4A1 was identified as a causative gene for hereditary porencephaly ${ }^{3}$. Variants in COL4A1 have been shown to be associated with a broad range of disorders, including small-vessel brain disease of various severities, including polencephaly, variably associated with eye defects (retinal arterial tortuosity, Axenfeld-Rieger anomaly, and cataract) and systemic findings (kidney involvement, muscle crumps, cerebral aneurysms, Raynaud phenomenon, cardiac arrhythmia, and hemolytic anemia) ${ }^{4}$.

To date, seven patients with a COL4A1-related disorder in association with hemolytic anemia have been reported. In 2013, Yoneda et al. reported that five among fifteen patients with COL4A1-related disorder had hemolytic anemia ${ }^{5}$, indicating that hemolytic anemia may be one of the related features of COL4A1-related disorder. Interestingly, five patients showed improvement of anemia, with the longest at 6 months of age. In 2016, Tomotaki et al. reported a male infant with COL4A1-related disorder with severe hemolytic jaundice. His anemia improved after the red cell transfusion on day 29 without recurrence ${ }^{6}$. Maisonneuve et al. reported a fetal case with severe anemia in association with cerebral ischemohemorrhagic damage revealed by ultrasonography, in whom a de novo COL4A1 mutation was identified ${ }^{7}$.

The clinical and hematological characteristics identified in this study are similar to those in previous reports ${ }^{5,6}$. The present four patients had severe jaundice and/or progressive anemia from birth, although there was no family history of anemia and jaundice. Three patients (Cases 2, 3, and 4) had icterus gravis neonatum and received phototherapy. Case 1 received blood transfusion seven times. These hematological features were almost certainly derived from COL4A1 mutations.

COL4A1 encodes COL4A1 ( $\alpha 1$ chain), which consists of an amino-terminal $7 \mathrm{~S}$ domain, a triple-helix forming collagenous domain, and a carboxyl-terminal noncollagenous domain. The triple-helix forming collagenous domain consists of glycine-X-Y amino acid repeats, which are essential for the formation of type IV collagen protein $^{8}$. COL4A1 forms a triple-helical structure with COL4A2 ( $\alpha 2$ chain) by combining as heterotrimers with a 2:1 stoichiometry (alpha1-alpha1-alpha2), forming the sheet-like network 9 .

The four COL4A1 variants identified in this study result in the substitutions of glycine for another amino acid residue in one of the glycine-X-Y repeats. The amino acid position of the mutation identified in Case 4, p.Gly948Asp, was the same as Case 4 reported by Yoneda et al., p. Gly948Ser, who did not show hemolytic anemia. The same amino acid of p.G785V identified in Case 1 was also affected in the case previously reported (p.G785E), with intrauterine stroke and anterior segment dysgenesis ${ }^{10}$.
The mechanisms of hemolytic anemia in patients with COL4A1-related disorder have not yet been established. However, some proposed explanations have been considered. One explanation is that dysfunction of basement membranes leads to red cell destruction through the vasculature or reticuloendothelial system and effective transmigration of red cells or erythroid progenitor cells. Janowska-Wieczorek et al. reported that peripheral blood CD34+ cells strongly express collagen type IV degrading gelatinases, matrix metalloproteinases-2 (MMP-2) and MMP- $9^{11}$. They suggested that type IV collagens expressed in bone marrow sinusoidal basement membranes influence the transmigration of blood progenitor cells. Infantile hemolytic anemia observed in four patients with COL4A1 variants spontaneously improved later. Morphological findings of the peripheral blood smear, such as schiozocytes and poikilocytes, were observed in peripheral blood smears in two patients. Transient hemolytic anemia and abnormal erythrocyte morphology resemble the findings of hereditary elliptocytosis during infancy. Transient hemolysis may depend on the development and growth of skeletal and nonskeletal red cell components.

It has been reported that free 2,3-diphosphoglycerate (DPG), presented in neonatal RBCs as a consequence of diminished binding to fetal $\mathrm{Hb}$, may render HE susceptible to in vivo fragmentation ${ }^{12}$. The developmental switch from fetal to adult hemoglobin, by diminishing available free 2,3-DPG, may explain the abatement of hemolytic anemia that accompanies maturation.

In conclusion, COL4A1-related disorder should be listed as one of the differential diagnoses when we encounter infants with undiagnosed hemolytic anemia and congenital malformations of the brain. We consider that permanent management for hemolytic anemia is not necessary for patients with COL4A1-related disorders. Further studies are required to clarify how COL4A1 mutations are involved in anemia.

\section{HGV database}

The relevant data from this Data Report are hosted at the Human Genome Variation Database at https://doi.org/10.6084/m9.figshare.hgv.2930, https://doi. org/10.6084/m9.figshare.hgv.2933, https://doi.org/10.6084/m9.figshare. hgv.2936, https://doi.org/10.6084/m9.figshare.hgv.2939.

\footnotetext{
Acknowledgements

We would like to express our gratitude to the patients and their parents for their cooperation. This work was supported by the Japan Agency for Medical Research and Development (AMED); the Project for Development of Innovative Research on Cancer Therapeutics (JP19cm0106501 to S.O.) and the Practical Research Project for Rare/Intractable Diseases (JP 19ek0109286h0003 to S.O.); the Ministry of Education, Culture, Sports, Science and Technology of Japan; the High Performance Computing Infrastructure System Research Project (hp160219, hp170227, hp180198, and hp190158 to S.O.) (this research used computational resources of the $\mathrm{K}$ computer provided by the RIKEN Advanced Institute for Computational Science through the HPCI System Research project); and JSPS KAKENHI (JP16K10041 to H.K.).
} 


\section{Author details}

'Department of Transfusion Medicine and Cell Processing, Tokyo Women's Medical University, Tokyo, Japan. ${ }^{2}$ Department of Pediatrics, Graduate School of Medical Sciences, Kyushu University, Fukuoka, Japan. ${ }^{3}$ Department of Neonatology, Musashino Red Cross Hospital, Tokyo, Japan. ${ }^{4}$ Department of Pediatrics, Shikoku Central Hospital, Ehime, Japan. ${ }^{5}$ Department of Hematology and Oncology, Shizuoka Children's Hospital, Shizuoka, Japan. ${ }^{6}$ Center of Advanced Medicine and Clinical Research, Nagoya University, Aichi, Japan. ${ }^{7}$ Department of Pathology and Tumor Biology, Kyoto University Graduate School of Medicine Faculty of Medicine, Kyoto, Japan. ${ }^{8}$ Institute for the Advanced Study of Human Biology (WPI-ASHBi), Kyoto, Japan. 'Department of Medicine, Center for Hematology and Regenerative Medicine, Karolinska Institute, Stockholm, Sweden. ${ }^{10}$ M\&D Data Science Center, Tokyo Medical and Dental University, Tokyo, Japan. "1Department of Pediatrics, Nagoya University Graduate School of Medicine, Nagoya, Japan. ${ }^{12}$ Institute of Medical Genetics, Tokyo Women's Medical University, Tokyo, Japan

\section{Author contributions}

All authors contributed to the research design, data acquisition, data analysis, data interpretation, drafting and revising the manuscript, and approval of the final version submission.

\section{Conflict of interest}

The authors declare that they have no conflict of interest.

\section{Publisher's note}

Springer Nature remains neutral with regard to jurisdictional claims in published maps and institutional affiliations.

Received: 31 July 2020 Accepted: 28 October 2020.

Published online: 27 November 2020

\section{References}

1. Hamada, M. et al. Whole-exome analysis to detect congenital hemolytic anemia mimicking congenital dyserythropoietic anemia. Int. J. Hematol. 108 306-311 (2018)

2. Richards, S. et al. Standards and guidelines for the interpretation of sequence variants: a joint consensus recommendation of the American College of Medical Genetics and Genomics and the Association for Molecular Pathology. Genet. Med. 17, 405-424 (2015).

3. Gould, D. B. et al. Mutations in Col4a1 cause perinatal cerebral hemorrhage and porencephaly. Science 308, 1167-1171 (2005).

4. Plaisier, E. \& Ronco, P. In GeneReviews $\left({ }^{\circledR}\right)$ (eds. Adam, M. P. et al.) (University of Washington, Seattle, 1993).

5. Yoneda, Y. et al. Phenotypic spectrum of COL4A1 mutations: porencephaly to schizencephaly. Ann. Neurol. 73, 48-57 (2013).

6. Tomotaki, S. et al. Severe hemolytic jaundice in a neonate with a novel COL4A1 mutation. Pediatr. Neonatol. 57, 522-525 (2016).

7. Maisonneuve, E. et al. Managing the unusual causes of fetal anemia. Fetal Diagn. Ther. 47, 156-164 (2020).

8. Kuo, D. S., Labelle-Dumais, C. \& Gould, D. B. COL4A1 and COL4A2 mutations and disease: insights into pathogenic mechanisms and potential therapeutic targets. Hum. Mol. Genet. 21, R97-R110 (2012).

9. Khoshnoodi, J., Pedchenko, V. \& Hudson, B. G. Mammalian collagen IV. Microsc. Res. Technol. 71, 357-370 (2008).

10. Durrani-Kolarik, S., Manickam, K. \& Chen, B. COL4A1 mutation in a neonate with intrauterine stroke and anterior segment dysgenesis. Pediatr. Neurol. 66, 100-103 (2017).

11. Janowska-Wieczorek, A. et al. Growth factors and cytokines upregulate gelatinase expression in bone marrow CD34(+) cells and their transmigration through reconstituted basement membrane. Blood 93, 3379-3390 (1999).

12. Mentzer, W. C. Jr. et al. Modulation of erythrocyte membrane mechanical stability by 2,3-diphosphoglycerate in the neonatal poikilocytosis/elliptocytosis syndrome. J. Clin. Invest. 79, 943-949 (1987). 